\title{
What survives theory change?
}

\begin{abstract}
To find out which properties of our actually most fundamental theories have reasonable chances to survive in more fundamental theories like quantum gravity or subquantum theories, we evaluate how similar properties tend to change or resist in case of theory changes between more fundamental theories and their approximations.

The most stable against theory change is space and time, followed by the configuration space. The laws of evolution are most vulnerable to theory change. A corresponding behavior can be seen for the corresponding symmetry groups, with translational symmetry being the most stable symmetry, and symmetries of evolution equations the most unstable symmetries.

We observe frequent changes between deterministic and stochastic theories, and identify general mechanisms as the cause for such changes: instabilities lead to deterministic chaos, while averages tend to follow deterministic laws. The consideration of a sound analogon of Lorentz symmetry suggests that a global symmetry which becomes localized in a more fundamental theory is doomed to disappear in the next more fundamental theory.
\end{abstract}

These results contradict popular opinions about local Lorentz- and gauge symmetries as well as quantum randomness having a deep, fundamental character.

Keywords: determinism, randomness, local lorentz symmetry, covariance, backgroundindependence
Volume I Issue 3 - 2018

Schmelzer I
Independent scientist, Germany

Correspondence: Schmelzer I, Independent scientist, Akeleiweg 7, 12487 Berlin, Germany, Email ilja.schmelzer@gmail.com

Received: April 23, 2018| Published: June 20, 2018

\section{Introduction}

I think, most of those interested in physics would like to understand what would be the most fundamental things. Unfortunately, we do not have an answer - we simply don't know. What we actually know are two "most fundamental" theories, and they are in deep conflict. Their conflict is mainly a conflict of their basic, fundamental properties, and what is considered essential, fundamental in one theory does not hold in the other one. Thus, if we restrict ourself to those properties shared by both, what remains is nothing. Moreover, there is Newton's "what we know is a drop, what we don't know is an ocean", and Popper's critical rationalism, which claims that we can never even be sure that our physical theories are true, even less that they are fundamentally true.

But for our search of unification we need some guidance, and to guess which of the properties of the existing theories surives in their unification, and what has to be given up, is the simplest and most natural way to get such guidance. So, there is more than simply metaphysical interest behind the question which of the properties of our fundamental theories are really fundamental, and have a good chance to remain valid at least in the next more fundamental theory, and which of them are only approximations.

On the other hand, there is already a long list of various approximations of our most fundamental theories, and we can use it to get some hints about which properties of theories tend to change almost always together with a theory change, and which properties remain more stable. The aim of this paper is to find out what information we can obtain in such an indirect way about which properties of our actual most fundamental theories have a good chance to survive, and which are almost doomed to fail.

A first observation is quite trivial. The most stable thing is space and time. The next more stable thing is the configuration space. The most unstable thing is the dynamics.

Indeed, the change of the theory of space and time is extremely rare, it happened only once, in the scientific revolution of relativity. The majority of modern physicists is sure that these insights into the non-trivial physical nature of space and time are fundamental, and expect further changes in near future, either based on quantum gravity or on string theory. But let's also note that the equations of special and general relativity do not force us to change our theory of space and time.

For special relativity, we have the Lorentz ether interpretation, which does not change the classical Newtonian ideas about space and time. All the relativistic effects are, in this interpretation, only distortions of our clocks and rulers, which, as a consequence, appear unable to measure true fundamental distances of space and true time.

And such a Lorentz ether interpretation exists for the Einstein equations of general relativity too, and appears surprisingly simple. We use the Einstein equations in harmonic coordinates, which are interpreted as the preferred Cartesian coordinates (what has been proposed already by Fock $^{1}$ ) and describe a classican Newtonian background of absolute space and time. The harmonic condition $\partial_{\mu}\left(g^{\mu v} \sqrt{-g}\right)=0$ define four conservation laws, which are interpreted as the classical continuity and Euler equations of standard condensed matter theory. This gives the following rules for defining density, velocity and stress tensor of the ether $\rho=g^{00} \sqrt{-g}, \rho v^{i}=g^{0 i} \sqrt{-g} \rho v^{i} v^{j}-\sigma^{i j}=g^{i j} \sqrt{-g}$. In particular $\partial_{\mu}\left(\mathrm{g}^{0 \mu} \sqrt{-g}\right)=\partial_{t} \rho+\partial_{i}\left(\rho v^{i}\right)=0$ becomes the continuity equation. The condition $\rho>0$ translates into the requirement that the preferred time coordinate has to be time-like. For more details see. ${ }^{2,3}$ So, we cannot 
even exclude completely that there is no change of the theory of space and time at all.

On the other hand, if the spacetime interpretation is correct, and the spacetime has really lost its special role as a stage, and became an actor itself, the special role of remaining stable and unchanged during theory change will plausible disappear too. So, despite the fact that Euclidean space has survived from the largest distances, the global structure of the universe, down to the smallest distances considered in quantum theory, we can expect some changes here in near future, and the result is completely open: From a spacetime of much higher dimension of string theory to some discrete structure of loop quantum gravity or a simple return of the classical special role as a stage in a Lorentz ether everything seems imaginable.

\section{Random or deterministic?}

A question which interests many people is if our world is truly random or deterministic. In particular, quantum theory is considered to be evidence that our universe is fundamentally random.

But, as we will see, if our actually most fundamental theory is a deterministic or stochastic theory does not give us much. To switch between determinism and randomness is surprisingly easy and happens quite often. So, to consider what our actually most fundamental theory tells us about determinism vs. randomness as a deep fundamental insight is not a good idea.

This will be already clear from observation of the actual situation. Quantum theory itself is a stochastic theory, and predicts only probabilities. But it has not only interpretations which present these probabilities as inherently random. Instead, there is also de Broglie-Bohm (dBB) theory, an interpretation which is fundamentally deterministic, and gives the quantum probabilities only in quantum equilibrium.

Similar switches seem to appear quite often. Let's start from the most fundamental theory and consider subsequent approximation. We can start with de Broglie-Bohm theory, even if this is yet a proposal for a subquantum theory. It is a deterministic theory. Then, in the quantum equilibrium, we obtain the stochastic predictions of quantum theory. The next step of approximation gives classical theory, with atoms following classical trajectories. In the next approximation, we are unable to see the atoms themselves, but can observe only larger pieces of matter, which are hit, in essentially random ways, by atoms. The result is Brownian motion of these larger particles - a stochastic movement. In the next steps, these small random influences become too small to be observable. All we can observe are averages. And these averages appear to follow deterministic equations of classical condensed matter theory and thermodynamics. And so it continues. The weather prediction is, essentially, stochastic: We can predict only probabilities for rain. But on a larger scale we have the climate, which is, again, deterministic. There are humans, and their behavior is often unpredictable. But then we have economic theory, which nonetheless predicts a lot about the results of human behavior.

So, the sheer number of examples already shows that such switches between deterministic and stochastic theories are quite common, so that we have to expect similar changes in future too. But it makes sense also to consider the mechanism which leads to such changes.

First, let's assume we have a deterministic theory. While a few things like energy may be conserved, these are exceptions, not the rule. In general some differences tend to decrease, others tend to increase. Both effects usually happen in an exponential form. As a consequence, after a short time even extremely small differences can have extremely large consequences. Such instabilities are, in general, unavoidable. But the consequence of these instabilities is that the future becomes effectively unpredictable, random. And to describe such random behavior we need stochastic theories.

What works in the other direction? Stochastic theories give us probabilities of various random events. But in the next step of approximation, to observe the particular random event becomes impossible, and all one can observe are average values. And there is the law of large numbers, which tells us that if the number of random events increases, the prediction of the average becomes more and more accurate. The consequence is that not only the particular average in the particular situation, but also the rules how the averages change in time, under the influence of external circumstances or influences described by other averages, become deterministic.

So, the switches between deterministic and stochastic theories are not accidental things, they happen regularly and have welldefined reasons, which are essentially always present. That means, the oscillations between deterministic and stochastic theories are predictable. And so we can expect that they will continue also if we switch to more fundamental, subquantum theories.

Of course, the explanation why the theory will switch worked only in one direction - approximation. This is simply because we have no possibility to derive a more fundamental theory from the existing one, even if we would like to have it. But so what? The prediction which follows is that there will be, over many steps of approximation, oscillations between deterministic and stochastic theories. And this property of the sequence of theories - oscillations between deterministic and stochastic theories - is direction-independent. So we can expect that it works in the other direction too.

We conclude that there is no reason at all to believe that considering the actually most fundamental theories will not help us to find an answer to the question "is the universe inherently random or deterministic". This question is, therefore, metaphysical.

\section{Symmetries}

Many of those who search for more fundamental theories value the symmetry groups of existing theory, in particular gauge symmetries and relativistic symmetry. But is it a good idea to bet that symmetry groups survive theory change?

First, different symmetries have to expect a different fate. What we have observed about the stability of space, configuration space and evolution equations naturally applies to the corresponding symmetry groups too. So the Euclidean symmetry of space has shown a similar stability as space, from the global universe down to quantum theory, and with a chance for recovery in the Lorentz ether.

As an example of failure we can use the group of canonical transformations. It is the symmetry group of the Hamilton formalism. That means, it is a symmetry group of the mathematical formalism of the evolution equations. The evolution in time has to be a canonical transformation. But it is clear that with a theory change the evolution equations will change, and their mathematical formalism will change too. So, it had no chance to survive in quantum theory. Quantum theory has evolution equations of a very different form, and so we 
have a very different corresponding symmetry group - unitary transformations. They are of the same type, so that they will also hardly survive in a subquantum theory.

In fact, canonical transformations make no sense in the Lagrange or Hamilton-Jacobi formalism of classical mechanics. So, symmetry groups of the mathematical formalism of the evolution equations are even more unstable than the physical theories with these evolution equations - they change already if the interpretation, the mathematical formalism, is changed. The fate of unitary symmetry is not better - in de Broglie-Bohm theory, even if this is only a particular interpretation of quantum theory, the symmetry is already different.

\section{What the sound variant of lorentz symmetry can tell us}

The most interesting symmetries are, of course, those considered to be fundamental today: Gauge symmetries and relativistic symmetry. Will they survive in quantum gravity or a theory of everything?

To study them, I propose to consider a theory which is mathematically quite similar to relativity - the analogon of Lorentz symmetry for sound waves of classical condensed matter theory. I think everybody has heard about the Doppler shift, with the classical example being the sound of the whistle of a passing train, which changes from high when the train comes to low when the train drives away. But much less known is a simple method how to transform a solution for the sound created by a whistle in rest into a solution for the sound created by a moving whistle: Apply to the solution the formula for a Lorentz transformation, only with the speed of sound instead of c. The reason for this is that such a sound version of Lorentz transformation is symmetry of the equation for sound waves $\square p(x, t)=\left(\mathrm{c}_{\text {sound }}^{-2} \partial_{t}^{2}-\Delta\right) p(x, t)=0$.

In this approximation, the sound waves are described as minor variations of a fixed background solution which has a constant density, constant pressure, and zero velocity. The size of the variation is too small to modify in an essential way density, velocity and pressure of the medium itself, so that we can use a fixed homogeneous static background solution as a reasonable approximation for the real solution. The resulting equation has global sound-Lorentz symmetry.

But this is, of course, only a very rough approximation. A more accurate and more fundamental description of sound waves has to consider also the possibility of inhomogeneities and deformations. What remains in this case from the original sound-Lorentz symmetry? Not much. But on a small scale one can continue to approximate the solution by a solution with constant pressure, velocity, and density. And at this small scale, one can use the local sound-Lorentz transformation with the same aim, to obtain at least locally a Dopplershifted solution. So, the global sound-Lorentz symmetry of the rough approximation survives as a local sound-Lorentz symmetry.

We also know what follows: Namely, in the next more fundamental theory, considering even smaller distances, we have atomic theory, and nothing survives from sound- Lorentz symmetry.

This sequence looks quite natural. What in the grossest approximation is a global symmetry, and transforms into a weaker, only approximate local symmetry in a less rough approximation, is not a good candidate for survival. It is already only an approximate symmetry, so that there is no reason to think that it is fundamental. So it has no chance for survival in the next step. We can make a preliminary conclusion: Our example of sound-Lorentz symmetry suggests that local Lorentz-invariance is something much weaker than the original global Lorentz-invariance of special relativity, and that in the next more fundamental theory it will probably disappear into nothing.

\section{About the difference between local sound- lorentz symmetry and general covariance}

Unfortunately, even if the symmetry group of GR is sometimes named "local Lorentz symmetry", it is something different than the local sound-Lorentz symmetry considered above. It is the group of all diffeomorphisms, much greater than the local sound-Lorentz symmetry of the wave equation in inhomogeneous media. What has happened here? The aim of this section is to understand the difference.

Roughly, we have to distinguish here three different things. First of all, every physical theory, even one without any nontrivial symmetry, can be described in a general covariant form. In this form, it seems to have diffeomorphism symmetry, but, given that a change in the formalism does not add symmetry to the physics, this is not a relevant symmetry. Then, there is the local Lorentz symmetry. And then, there is yet another thing, background independence. These three things are quite different, as we want to show in this section.

\section{The kretschmann objection}

That general covariance has no physical content is an objection made by Kretschmann. ${ }^{4}$

Einstein accepted this point in his answer. ${ }^{5}$ His counterargument was that, even if one can do it, the resulting covariant version of Newtonian theory would be so complex and ugly that it would not have a chance in comparison with simple and beautiful GR. This was done in Newton-Cartan theory, ${ }^{6}$ which looked not really horribly ugly. But this is quite irrelevant for us - what matters here are that general covariance is nothing which describes a physical symmetry of the theory. However asymmetric a theory is, we can construct a general covariant formulation. And however ugly the result, the symmetry group of the covariant equations will be the full group of diffeomorphisms. Does this tell us something about the theory being symmetric? Obviously not. A general covariant formulation does not make a theory more symmetric.

\section{The lorentz ether interpretation of the einstein equations}

The Lorentz interpretation of the Einstein equations of $\mathrm{GR}^{3}$ we have described above seems helpful to illustrate this.

It is clear that a general inhomogeneous metric configuration of the ether does not have any nontrivial symmetry. Now let's see how we make this interpretation covariant. We recognize that the preferred coordinates are functions on the background, which would be normal scalar functions if described in other, general coordinates. We denote these functions by $\mathfrak{x}^{\mu}(x)$. Then, we recognize that the continuity and Euler equations can be rewritten in terms of the "metric" $\mathrm{g}_{\mu \nu}$ defined by the formulas above, and appear, then, equivalent to covariant equations for the preferred coordinates $\square \mathfrak{x}^{\alpha}(x)=\left(\partial_{\mu} \mathrm{g}^{0 \mu} \sqrt{-g} \partial_{v}\right) \mathfrak{x}^{\alpha}(x)=0$. The other equations are simply the Einstein equations, thus, already covariant. So, we have found for the inhomogeneous Lorentz ether, which does not have any symmetry for a general configuration, a covariant set of equations. 
This is, obviously, a symmetry of the mathematical formalism only, and not a symmetry of the physical theory.

But let's also note that the Lorentz ether interpretation is physically different from GR in the spacetime interpretation: It has a fixed background, thus, only topologically trivial solutions. And the preferred time is a global time-like function, so that the solutions have to be global hyperbolic. This excludes wormholes as well as causal loops like Gödel's rotating universe.

To summarize: This Lorentz ether interpretation differs from GR because it has a Newtonian background, thus, is not backgroundindependent. It has, like GR, local Lorentz symmetry as a physical symmetry. And it has a non-covariant formulation in preferred coordinates, but also a covariant formulation with a corresponding formal diffeomorphism symmetry.

\section{The einstein aether}

And there is another example, the "Einstein aether" proposed by Jacobson and Mattingly. ${ }^{7}$ This is a theory which, together with the metric, contains in every point a vector field - the direction of movement of the "Einstein aether". Different from the Lorentz interpretation of the Einstein equations of GR, there is no global Newtonian background in this theory. The theory is, by construction, background-independent. The preferred direction in each point does not combine into a global preferred direction. In particular, there are also Gödel-like solutions with causal loops for the Einstein aether. ${ }^{8}$

Nonetheless, in each point we have a well-defined preferred frame, and therefore no local Lorentz invariance. So, we have background independence and full diffeomorphism symmetry, but no local Lorentz invariance.

To summarize, one has to distinguish three different things: general covariance (which is irrelevant for symmetry), local Lorentz invariance (present in the Lorentz ether interpretation but not in the Einstein aether) and background independence (present in GR and the Einstein aether but not in the Lorentz ether interpretation).

General covariance is only a technique applicable to arbitrary classical theories, and therefore irrelevant for the symmetry of physics. Background independence makes, instead, a physical difference, but the difference has nothing to do with Lorentz symmetry. If it survives is a completely different question, and the consideration of other theories from the history of physics will not tell us anything about this. So, our argument based on the sound analogon of the Lorentz symmetry applies only to local Lorentz invariance.

But for this particular aspect of relativistic symmetry, the argument seems quite strong. Namely, local Lorentz symmetry appears weaker than global Lorentz symmetry: Global Lorentz symmetry allows to construct new exact Doppler-shifted global solutions out of existing ones, while local Lorentz symmetry does not have this power to construct global exact Doppler-shifted solutions for a given, fixed metric. So, local Lorentz symmetry is an already essentially weakened variant of the originally stronger global Lorentz symmetry, and therefore seems doomed.

\section{Conclusion}

Let's summarize what we have found by considering various examples of approximate theories with the aim to find out what often changes if we switch to a more fundamental theory and what has a chance to survive.
If a theory is deterministic or stochastic does not really matter - this changes in a very fast way from theory to theory, with deterministic chaos and laws of large numbers as the mechanisms which allow to switch forth and back between determinism and randomness.

The most stable thing is space and time, followed by the configuration space, with the evolution equations being most unstable. A similar fate have the corresponding symmetry groups, but they additionally appear to depend even on interpretations. Euclidean symmetry of space appeared to be the most stable symmetry, with the spacetime interpretation of GR being the only exception. Instead, canonical transformations, present only in a particular formalism of classical mechanics, is a negative example.

The analogon of Lorentz symmetry for sound waves has suggested that if a global symmetry becomes localized in a first step, it will disappear completely in the next step. This conclusion would be in strong contradiction with the strong belief that relativistic and gauge symmetries are fundamental insights which remains forever.

To clarify if the example of local sound-Lorentz symmetry is really comparable with the symmetry of GR appeared to be surprisingly complicate. We had to distinguish the local Lorentz symmetry from background independence as well as from the formal diffeomorphism symmetry which follows from a covariant formulation. The consideration of Kretschmann's objection and the examples of the Einstein aether and the Lorentz ether interpretation of the Einstein equations have helped us to clarify this. The argument we have found considering the sound analogon is applicable to local Lorentz invariance, but remains silent about background independence.

\section{Acknowledgements}

None.

\section{Conflict of interest}

The author declares that there is no conflict of interest.

\section{References}

1. Fock V. The Theory of Space Time and Gravitation. Pergamon Press, Oxford. 1964. $460 \mathrm{p}$

2. Schmelzer I. A generalization of the Lorentz ether to gravity with general-relativistic limit. Advances in Applied Clifford Algebras. 2012;22(1):203-242.

3. Schmelzer I. Ether Interpretation for the Einstein Equations of General Relativity. In: Reimer A. editor. Horizons in World Physics. Nova Science Publishers. 2017;294

4. Kretschmann E. Über den physikalischen Sinn der Relativitätspostulate A Einsteins neue und seine ur-sprüngliche Relativititstheorie. Ann Phys. 1917;53:575-614.

5. Einstein A. Prinzipielles zur Allgemeinen Relativitätstheorie. Ann Phys 1918;55:241-244.

6. Cartan E. Sur les varéés à connexion affine et la théorie de la relativit géréraliće(premère partie). Ann Ecole Norm. 1923;40:325.

7. Jacobson T, Mattingly D. Gravity with a dynamical preferred frame. Phys Rev D. 2001

8. Gürses M. Godel Type Metrics in Einstein-Aether Theory. General Relativity and Quantum Cosmology. 2008. 